\title{
CT Scan Features Of Non Epithelial Ovarian Cancer - A Retrospective Study In Radiodiagnostic Department Of Dr. Soetomo General Hospital Surabaya At January 2016 - July 2019
}

\author{
H. Situmeang ${ }^{\mathrm{a}}$, L. Mardiyana ${ }^{\mathrm{b}}$, B. Soeprijanto ${ }^{\mathrm{c} *}$ \\ ${ }^{a}$ helfrdasitumeang@gmail.com \\ ${ }^{a}$ Resident of Radiology Department, Faculty of Medicine Universitas Airlangga/Dr.Soetomo General Hospital, Surabaya, Indonesia \\ ${ }^{b, c}$ Staff of Radiology Department, Faculty of Medicine Universitas Airlangga/Dr.Soetomo General Hospital, Surabaya, \\ Indonesia
}

\begin{abstract}
Ovarian carcinoma is the second most common gynecological malignancy and in Indonesia it is the $5^{\text {th }}$ most common cause of death in America, and in Indonesia it ranks 3rd after breast and cervix. Non-epithelial ovarian carcinoma is very rare and is often diagnosed at an advanced stage. CT scan is an imaging for diagnostic that can show a profile of non-epithelial ovarian carcinoma and this has never been studied in Dr. Soetomo General Hospital. The objective of this study is to evaluate solid component, cystic, fat, calcified, enhancement, unilateral / bilateral locations on CT scans of non-epithelial ovarian carcinoma patients. From January 2016 until July 2019, 55 patients of epithelial ovarian cancer with histopathological proven (age range $08-70$ years) were enrolled in the study. Patient did CT scan before operation. CT scan was evaluated in raw data. Based on age category, age range was $08-70$ years. $43.6 \%$ of patients are in the age range 41-60 years. CT scan features of non-epithelial ovarian cancer at Dr. Soetomo General Hospital on Germ Cell Tumor type 28 cases (50.9\%), Sex Cord Stromal Tumor 26 people (47.3\%), Tumor metastasis 1 case $(1.8 \%)$. Solid degenerative changes, necrosis, bleeding, and irregular contours were found in $85.5 \%$ of cases. Cystic features were found in $85.5 \%$ of cases, $14.5 \%$ of cases had no cystic component. Fat features were found in $23.6 \%$ of cases. Calcification features were found in $72.7 \%$ of cases. Enhancement was found in $98.2 \%$ of cases. As many as $96.4 \%$ of cases were found in unilateral. The CT scan feature of the germ cell tumor contained solid irregular components, enhancement, and unilateral, as many as $85.7 \%$ of cases had the cystic component, fat density and calcification were obtained in mature and immature teratomas. Sex cord stromal tumors have an irregular solid component and enhancement (enhancement), as many as $84.6 \%$ of cases with a cystic component, $83.3 \%$ of cases of unilateral.Tumor metastasis shows a unilateral appearance of a cystic mass with solid component.
\end{abstract}

Published by IJRP.ORG. Selection and/or peer-review under responsibility of International Journal of Research Publications (IJRP.ORG)

\section{Background}

Gynecological malignancies include cervical carcinoma, endometrial carcinoma and ovarian carcinoma. Ovarian carcinoma is the second most frequent of gynecological malignancies. Ovarian carcinoma is the deadliest gynecological malignancy. Fewer than half of patients survive more than five years after being diagnosed (Doubeni et al., 2016).

Ovarian carcinoma is the fifth leading cause of cancer death in women in the United States, after lung, breast, colorectal and pancreatic carcinoma, contributing to more than $3 \%$ of all carcinomas in women and the highest cause of death compared to other gynecological malignancies (Kang et al., 2018). The incidence of ovarian carcinoma increased by more than 30\% in the last decade, while the mortality due to ovarian carcinoma increased by $18 \%$. It is estimated that one in 70 women will develop ovarian carcinoma and 1 in 100 women will die from the disease. The highest incidence of ovarian carcinoma is highest in North America and Northern Europe and next in Japan (Fortsner R, 2019). In Indonesia, ovarian carcinoma ranks 3rd with an incidence rate of $7.07 \%$ after cervical and breast carcinoma in women of all ages.

More than $75 \%$ of ovarian carcinomas are diagnosed at an advanced stage and have metastases because at an early stage it is often asymptomatic and symptoms at an advanced stage are often non-specific. This causes a higher risk of death than endometrial carcinoma or cervical carcinoma. Early diagnosis of tumor lesions that are small and confined to the ovary is the most important prognostic (Doubeni et al., 2016; Hebbar et al.,2015). 
CT scan of the abdomen and pelvis is the first-line imaging modality used for preoperative staging. The goal of imaging is to help diagnose and differentiate malignant and benign tumors and to determine the staging of ovarian carcinoma. Ovarian carcinoma staging is a basic principle and one of the most important first steps to predict the patient's prognosis and plan the most appropriate treatment. As a preoperative imaging staging CT scan can present the difference between a patient requiring cytoreduction or a patient requiring neoadjuvant chemotherapy prior to surgery (Sahdev A, 2016).

\section{Methods}

This research is a descriptive type with a retrospective study design. This research at the Diagnostic Radiology Installation of the Integrated Diagnostic Center Dr. Soetomo Surabaya during January 2016 - July 2019. The sample collection in this study was carried out by consecutive sampling on all clinical patients with suspicious ovarian tumors who were subjected to abdominal CT scans with contrast and histopathological examinations had been carried out at Dr. Soetomo Surabaya.

The inclusion criteria in this study were patients with ovarian carcinoma who underwent a CT scan at Dr. Soetomo before surgery and a patient with ovarian carcinoma with histopathological results of non-epithelial ovarian carcinoma surgery

The research results are presented descriptively in tables and diagrams. The study was conducted after obtaining approval of an ethical test by the Medical Research Ethics Committee of Universitas Airlangga / Dr. Soetomo General Hospital Surabaya.

\section{Results}

The total number of research samples was 55 people. Non-epithelial ovarian carcinoma patients who have undergone a CT scan have the youngest age of 8 years and the oldest age of 70 years. Most patients with non-epithelial ovarian carcinoma were at the age of 50 years. The mean age of patients with non-epithelial ovarian carcinoma was $43.07 \pm 16.3$ years.

The results showed that most ovarian carcinoma patients were in the age group 41-60 years, $43.6 \%$ (24 people), 30.9\% (17 people) in the age group in the 21-40 year age group, 16.4\% ( 9 people) in the 61-80 years age group and at least in the 120 years age group $9.1 \%$ ( 5 people)

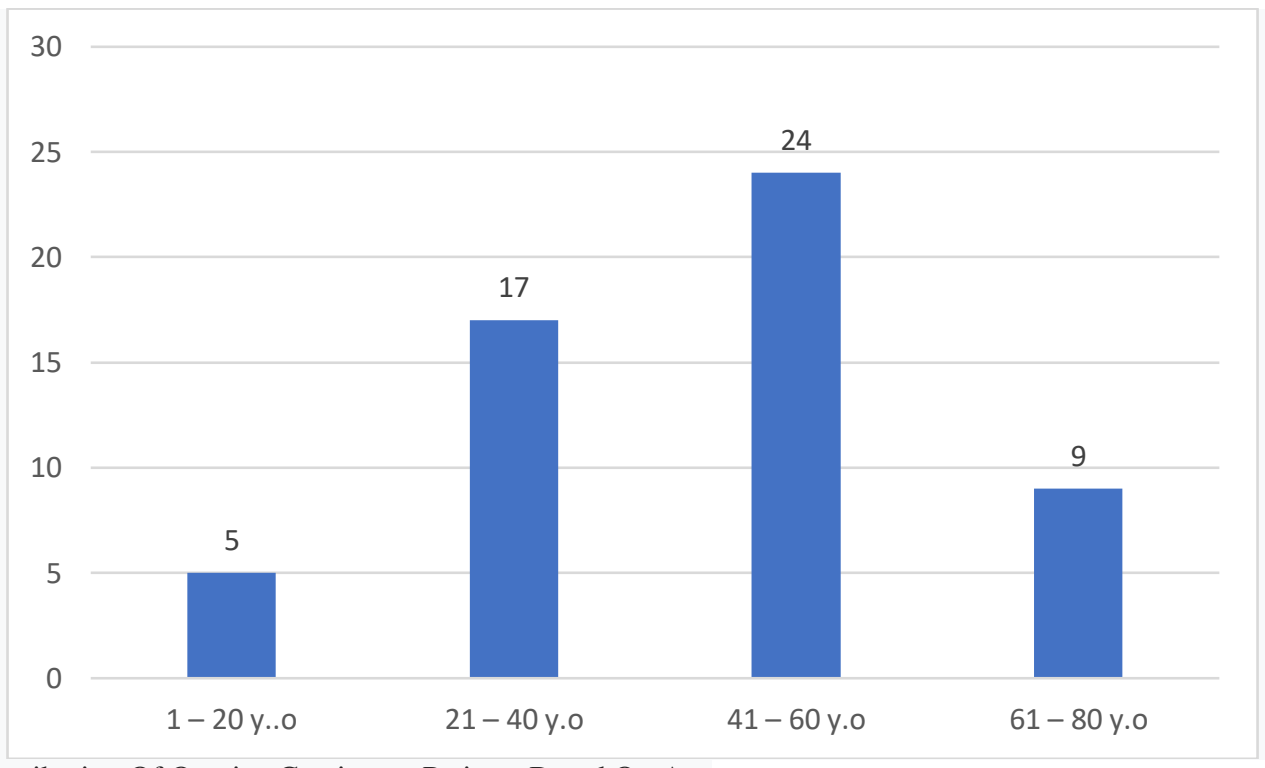

Figure 1. Distribution Of Ovarian Carcinoma Patients Based On Age

\subsection{CT Scan Feature of Non Epithelial Ovarian Carcinoma}

The CT scan features observed included solid, cystic, fatty, calcification, enhancement, unilateral / bilateral site components of 55 non-epithelial ovarian carcinoma patients.

The age group of 1 - 20 years has a germ cell tumor type non-epithelial ovarian carcinoma. The age group of 21-40 years has the most number of germ cell tumor-type non-epithelial ovarian carcinomas. Whereas in the age group $41-60$ years the most with non-epithelial ovarian carcinoma type sex cord stromal tumor.

A solid component of the CT image of patients with epithelial ovarian carcinoma with the type of degenerative changes, necrosis, bleeding, and irregular contours is most commonly found in non-epithelial germ cell tumor ovarian carcinoma. Homogeneous components and regular contours were also found in this study and mostly in the stromal sex cord tumors. Cystic feature on

CT scans of 55 patients with non-epithelial ovarian carcinoma were found mostly in non-epithelial germ cell tumor ovarian carcinoma. Fat features are found in non-epithelial germ cell tumor ovarian carcinoma. There is no fatty component in non-epithelial sex cord ovarian carcinoma, tumor or metastasis. The appearance of calcification was found mostly in nonepithelial germ cell tumor ovarian carcinoma and the most negative calcification in stromal sex cord tumors, did not appear calcification in metastatic non-epithelial ovarian carcinoma.

Contrast enhancement was found in almost all non-epithelial ovarian carcinoma patients, namely 54 patients from 55 study samples (98.2\%) according to the sample that had a solid component so that contrast showed enhancement.

Table 1. Cross-tabulation between age groups, CT images with non-epithelial ovarian carcinoma types (Germ Cell Tumor, 
Sex Cord Stromal Tumor, Metastasis)

\begin{tabular}{|c|c|c|c|c|c|}
\hline \multicolumn{3}{|c|}{ Variable } & Germ Cell Tumor & Sex cord stromal & Metastasis \\
\hline \multirow[t]{4}{*}{ Based of Age } & & $1-20$ years old & $3(60 \%)$ & $2(40 \%)$ & $0(0 \%)$ \\
\hline & & $21-40$ years old & $13(76,5 \%)$ & $4(23,5 \%)$ & $0(0 \%)$ \\
\hline & & $41-60$ years old & $8(33,3 \%)$ & $15(62,5 \%)$ & $1(4,2 \%)$ \\
\hline & & $61-80$ years old & $4(44,4 \%)$ & $5(55,6 \%)$ & $0(0,0 \%)$ \\
\hline \multirow{13}{*}{$\begin{array}{l}\text { CT Scan } \\
\text { feature }\end{array}$} & Solid with hon & nous and regular contour & $1(20 \%)$ & $4(80 \%)$ & $0(0,0 \%)$ \\
\hline & No solid comp & & $3(100 \%)$ & $0(0,0 \%)$ & $0(0,0 \%)$ \\
\hline & $\begin{array}{l}\text { Solid with deg } \\
\text { bleeding, i }\end{array}$ & $\begin{array}{l}\text { ative changes, necrotic, } \\
\text { lar contour }\end{array}$ & $24(51,1 \%)$ & $22(46,8 \%)$ & $1(2,1 \%)$ \\
\hline & Cystic & Positive & $24(51,1 \%)$ & $22(46,8 \%)$ & $1(2,1 \%)$ \\
\hline & & Negative & $4(50 \%)$ & $4(50 \%)$ & $0(0,0 \%)$ \\
\hline & Fat & Positive & $13(100 \%)$ & $0(0,0 \%)$ & $0(0,0 \%)$ \\
\hline & & Negative & $15(35,7 \%)$ & $26(61,9 \%)$ & $1(2,4 \%)$ \\
\hline & Calcification & Positive & $13(86,7 \%)$ & $2(13,3 \%)$ & $0(0,0 \%)$ \\
\hline & & Negative & $15(37,5 \%)$ & $24(60,0 \%)$ & $1(2,5 \%)$ \\
\hline & Enhancement & Positive & $27(50,0 \%)$ & $26(48,1 \%)$ & $1(1,9 \%)$ \\
\hline & & Negative & $1(100,0 \%)$ & $0(0,0 \%)$ & $0(0,0 \%)$ \\
\hline & Site & Unilateral & $27(50,9 \%)$ & $25(47,2 \%)$ & $1(1,9 \%)$ \\
\hline & & Bilateral & $1(50,0 \%)$ & $1(50,0 \%)$ & $0(0,0 \%)$ \\
\hline
\end{tabular}

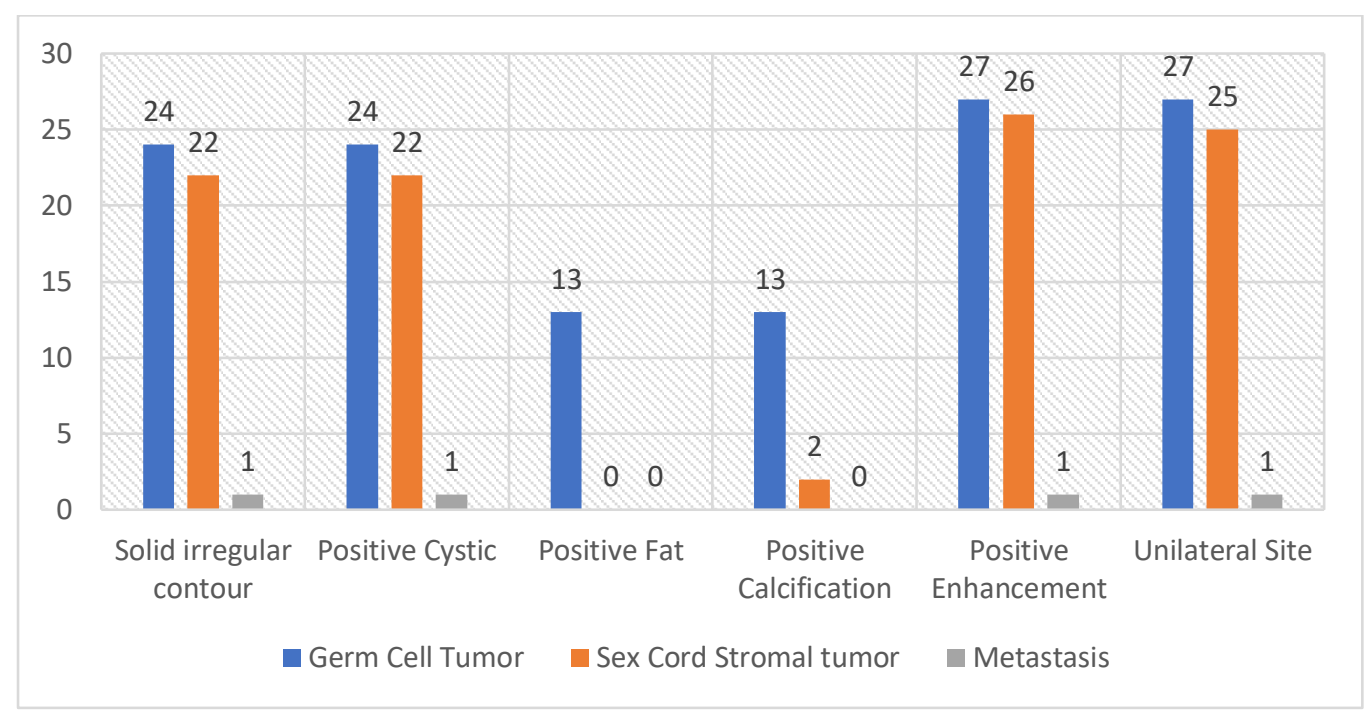

Figure 2. Diagram of CT scan feature of Non Epithelial Ovarian Carcinoma

\subsection{Distribution of Ovarian Carcinoma}

Non-epithelial ovarian carcinomas include germ cell tumors, sex-cord stromal tumors and metastases. The results showed that the majority of non-epithelial germ cell tumor ovarian carcinoma patients were 50.9\% (28 cases), non-epithelial sex-cord stromal ovarian carcinoma was $47.3 \%$ (26 cases) with metastatic non-epithelial ovarian carcinoma $1.8 \%$ ( 1 case).

Table 2. Distribution Based On Non Epithelial Ovarian Carcinoma Types

\begin{tabular}{lcc}
\hline Non Epithelial Ovarian Carcinoma Types & Frequency & Percentage $(\%)$ \\
\hline Germ cell tumor & 28 & 50,9 \\
Sex cord stromal tumor & 26 & 47,3 \\
Metastasis & 1 & 1,8 \\
\hline Total & 55 & 100 \\
\hline
\end{tabular}

Table 3. Distribution Based On The Type Of Non Epithelial Ovarian Carcinoma Germ Cel Tumor

\begin{tabular}{lcc}
\hline Germ Cell Tumor Types & Frequency & Percentage (\%) \\
\hline Mature Cystic Teratoma & 12 & 42,8 \\
Immature Teratoma & 7 & 25,0 \\
Dysgerminoma & 3 & 10,7 \\
\hline
\end{tabular}




\begin{tabular}{lcc}
\hline Yolk Sac Tumor & 5 & 17,9 \\
Non Gestational Choriocarcinoma & 1 & 3,6 \\
Embryonal carcinoma, Polyembryoma \& Mixed Germ Cell & 0 & 0,0 \\
Tumor & & 100 \\
\hline Total & 28 & 1 \\
\hline
\end{tabular}

Table 4. Distribution Based On The Type Of Non Epithelial Ovarian Carcinoma Sex Cord Stromal Tumor

\begin{tabular}{lcc}
\hline Sex Cord Stromal Tumor Types & Frequency & Percentage (\%) \\
\hline Adult Granulosa Cell Tumor & 15 & 57,8 \\
Juvenile Granulosa Cell Tumor & 1 & 3,8 \\
Fibroma ovarii & 5 & 19,2 \\
Cellular Fibroma & 1 & 3,8 \\
Sertoli Cell Tumor & 1 & 3,8 \\
Sertoli Leydig Cell Tumor & 1 & 3,8 \\
Sex cord Stromal Tumor, NOS & 2 & 7,7 \\
Fibrosarcoma & 0 & 0,0 \\
Thecoma & 0 & 0,0 \\
Luteinized Thecoma associated with sclerosing peritonitis & 0 & 0,0 \\
Slerosing Stromal Tumor & 0 & 0,0 \\
Signet ring stromal Tumor & 0 & 0,0 \\
Microcystic Stromal Tumor & 0 & 0,0 \\
Steroid Cell Tumor & 0 & 0,0 \\
Sex cord Tumor with annular tubules & 0 & 0,0 \\
\hline Total & $\mathbf{2 6}$ & $\mathbf{1 0 0}$ \\
\hline
\end{tabular}

\section{Discussion}

This research is a descriptive study with a retrospective research design that uses medical record data and raw data in the form of CT scan data at Diagnostic Centre Dr. Soetomo General Hospital Surabaya. Subjects were taken using consecutive sampling, in which each patient who met the inclusion criteria was included in the study during the specified time. The number of samples in this study amounted to 55 people.

Patients with non-epithelial ovarian carcinoma who underwent a CT scan were 8 years old and 70 years old. Most ovarian carcinoma cases were at the age of 50 years and the age range of 41-60 years as many as 24 cases (43.6\%). Non-epithelial ovarian carcinoma is often diagnosed in girls and women of childbearing age.

The highest number of non-epithelial ovarian carcinomas was found in Germ Cell tumors, namely 28 cases $(50.9 \%)$, then Sex Cord Stromal tumors 26 cases $(47.27 \%)$ and 1 case with tumor metastases (1.8\%). GCT is the second largest group of ovarian carcinoma after epithelial ovarian carcinoma (Shaaban et al., 2014).

Of the 28 cases with GCT, the most were found in the age range of 21-40 years, namely 13 cases (23.6\%), and of the 26 cases with Sex Cord Stromal Tumors the most were found in the age range 41-60 years as many as 15 people (27, 3\%). GCT is mainly diagnosed in young women and the first 3 decades and SCST occurs in women of all age ranges but generally in the fifth and sixth decades (Boussios $\mathrm{S}$ et al., 2016)

From a total of 55 research samples, solid components (degenerative changes, necrosis, bleeding, irregular contours) on the CT scan images of non-epithelial ovarian carcinoma patients were found in most cases, as many as 47 cases $(85.5 \%)$ and the most common was ovarian carcinoma. non epithelial germ cell type tumor. The solid component (homogeneous, regular contour) was found in 5 cases $(9.1 \%)$ and most of the non-epithelial ovarian carcinoma type sex cord stromal tumor, namely ovarian fibroma. CT scan of the fibroma appears as a solid mass and homogenous contrast enhancement (Horta M et al., 2015) Malignant ovarian tumors have a solid component with irregular contour, hemorrhage, necrosis, and degenerative changes. In this study, there was a solid irregular contour and necrosis, no solid degenerative changes or bleeding. Benign ovarian tumors are characterized by a homogeneous solid component with regular contours.
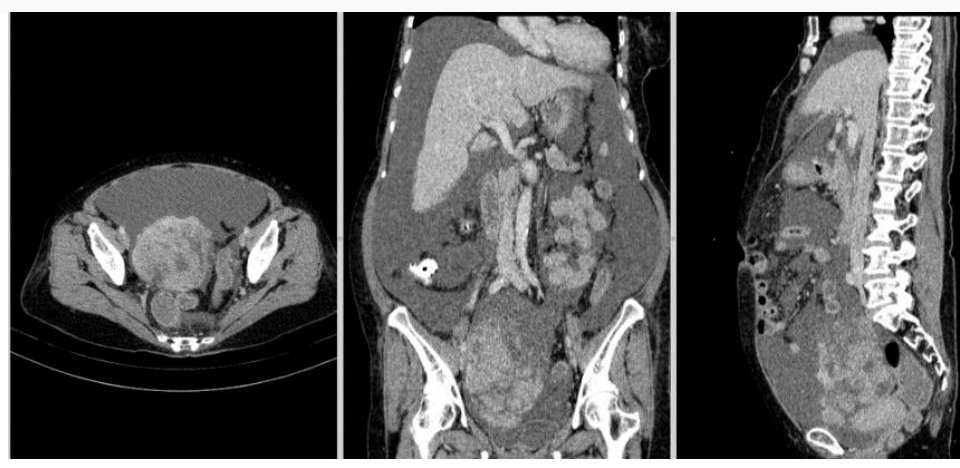

Figure 3. 59 years old female with adult granulosa cell tumor. CT scan shows solid irregular feature 
From a total of 55 study samples, cystic features were found in all non-epithelial ovarian carcinomas Germ Cell Tumors, as many as 28 cases (50.9\%), followed by Sex cord stromal tumors in 26 cases $(47.3 \%)$ and in tumor metastases 1 case $(1,8 \%)$. Non-epithelial ovarian carcinoma, apart from being dominant with a solid component, can also be found with a cystic component or a dominant cystic component with a solid component.
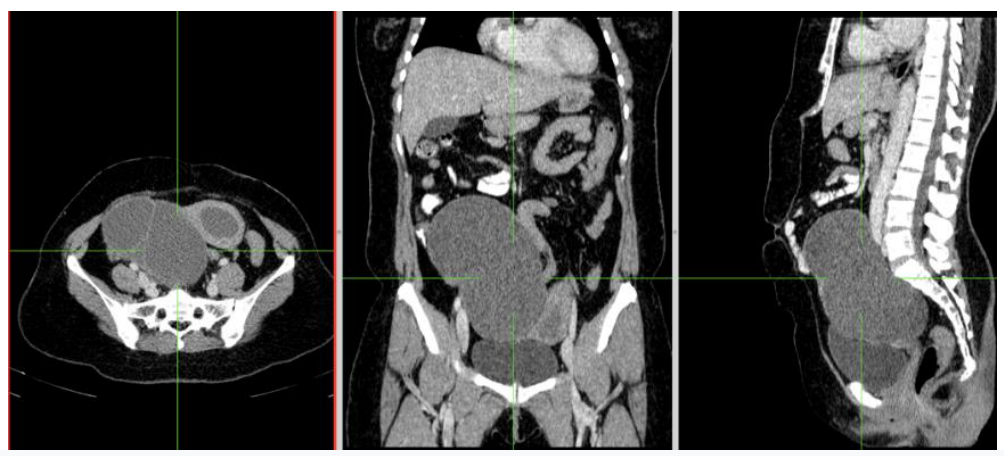

Figure 4. 41 years old female with mature cystic teratoma. CT scan shows a cystic appearance

From a total of 55 study samples, fat image was only found in non-epithelial germ cell tumor ovarian carcinoma, namely 13 cases $(23.6 \%)$, there was no fat component in the sex cord stromal tumor or in tumor metastasis. This is because fat is only found in immature and mature teratoma Germ Cell tumors. It is in accordance with the literature that in addition to mature teratoma, ovarian tumors with a fat component are immature teratomas (Jeong et al., 2000). Mature cystic teratomas and immature teratomas consist of tissues derived from germ cells: ectoderm, mesoderm and endoderm (Shaabaan et al., 2014). On CT scan a mass of fat density within a cystic mass with or without calcification on the wall leads to a mature cystic teratoma and on CT scan the presence of fat or calcification is a feature of the teratoma mass (Forstner R, 2019).
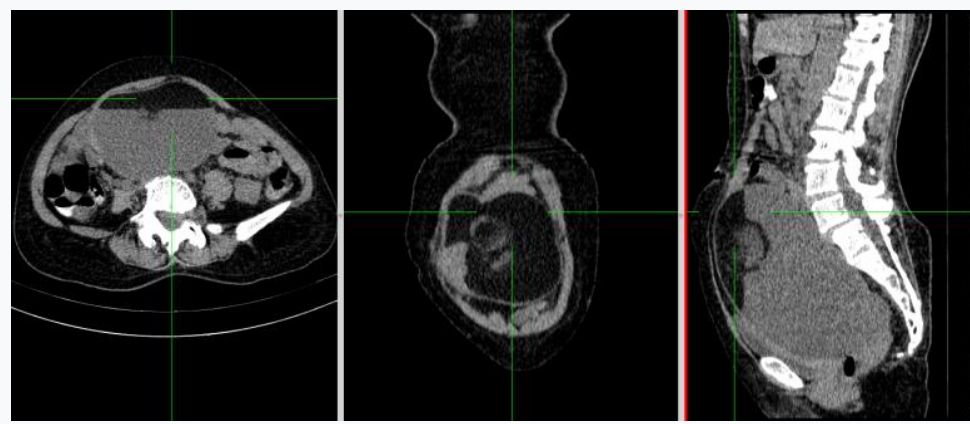

Figure 5. 60 years old female with mature teratoma. CT scan shows fat appearance

From a total of 55 research samples, the most common figures for calcification were found in non-epithelial ovarian carcinoma Germ Cell Tumors, namely 13 cases (23.6\%), there was a calcification component in the Sex cord stromal tumor as many as 2 cases (3.6\%). calcification in tumor metastases. This is because calcification is mainly found in immature and mature teratoma-type Germ Cell tumors, according to the literature that on CT scans the presence of fat or calcification is a feature of the teratoma mass (Forstner R et al., 2016).
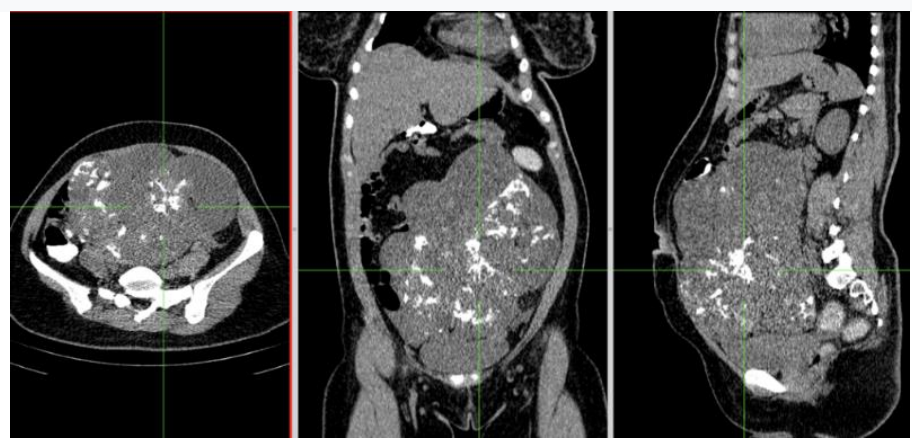

Figure 6. 33 years old female with immature teratoma. CT scan shows images of calcification

From a total of 55 research samples, an enhancement image was found in non-epithelial ovarian carcinoma Germ Cell Tumor, 27 cases $(49.1 \%)$, there was an image of enhancement in all stromal sex cord tumors, 26 cases (47, 3\%), followed by tumor metastasis in 1 case (1.8\%). This is because non-epithelial ovarian carcinoma is a dominant solid lesion compared to other types of ovarian carcinoma, the solid component of the tumor will appear to increase enhancement on contrast 
administration.

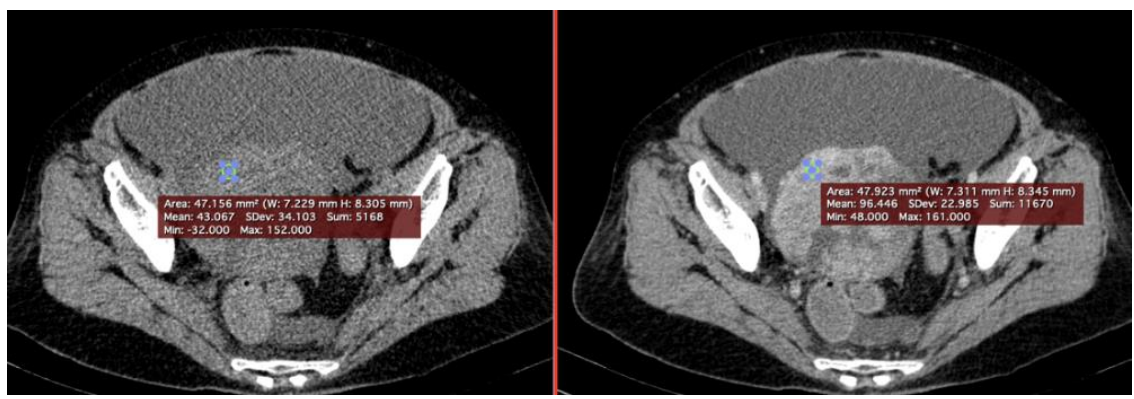

Figure 7. 59 years old female with adult granulosa cell tumor. CT scan shows the feature of enhancement

\section{Conclusion}

From the results of a CT scan study of non-epithelial ovarian carcinoma at dr. Soetomo found that all cases of ovarian carcinoma of the type of Germ Cell Tumor gave a heterogeneous mass picture where there was a solid irregular picture, enhancement and unilateral. As many as $85.7 \%$ of cases had a cystic component, except for choriocarcinoma, $30 \%$ of cases of dysgerminoma and $30 \%$ of cases of yolk sac tumor. Fat density was found in $83.3 \%$ of cases of mature cystic teratoma and $71.4 \%$ of cases of immature teratoma, and calcification in $66.6 \%$ of cases of mature cystic teratoma and $85.7 \%$ of cases of immature teratoma. All cases of Sex cord stromal tumors have a solid irregular appearance and enhancement. $84.6 \%$ of cases had a cystic component, except for Cellular fibroma, Sertoli cell tumors and $60 \%$ of cases of ovarian fibroma. All cases were found unilateral except in $6.7 \%$ of adult granulosa cell tumor cases which were bilateral. Tumor metastasis in this study was only found in 1 case and showed a picture of a cystic mass accompanied by an enhanced and unilateral solid component.

\section{Acknowledgment}

I would like to dedicate my gratitude to all the lecturers of Radiology, Faculty of Medicine Universitas Airlangga, my family, and my friends for their endless support.

\section{Conflicts of interest}

The author declares that there is no conflicts of interest

\section{References}

Doubeni, C.A., Doubeni, A.R.B., Myers, A.E., 2016. Diagnosis and management of ovarian cancer. Am. Fam. Physician 93, 937-944. https://doi.org/10.1080/21548331.1987.11707701

Kang, S.K., Reinhold, C., Atri, M., Benson, C.B., Bhosale, P.R., Jhingran, A., Lakhman, Y., Maturen, K.E., Nicola, R., Pandharipande, P. V., Salazar, G.M., Shipp, T.D., Simpson, L., Small, W., Sussman, B.L., Uyeda, J.W., Wall, D.J., Whitcomb, B.P., Zelop, C.M., Glanc, P., 2018. ACR Appropriateness Criteria ® Staging and Follow-Up of Ovarian Cancer. J. Am. Coll. Radiol. 15, S198-S207. https://doi.org/10.1016/j.jacr.2018.03.015

Forstner, R., 2019. CT and MRI in ovarian carcinoma. Med. Radiol. 287-323. https://doi.org/10.1007/174_2017_17

Hebbar, S., Moideen, N., 2015. Imaging in ovarian Cancer. Int. J. Reprod. Contraception, Obstet. Gynecol. 4, 1. https://doi.org/10.5455/2320-1770.ijrcog20150201

Sahdev, A., 2016. CT in ovarian cancer staging: How to review and report with emphasis on abdominal and pelvic disease for surgical planning. Cancer Imaging 16, 1-9. https://doi.org/10.1186/s40644-016-0076-2

Shaaban, A.M., Rezvani, M., Elsayes, K.M., Baskin, H., Mourad, A., Foster, B.R., Jarboe, E.A., Menias, C.O., 2014. Ovarian malignant germ cell tumors: Cellular classification and clinical and imaging features. Radiographics. https://doi.org/10.1148/rg.343130067

Boussios, S., Zarkavelis, G., Seraj, E., Zerdes, I., Tatsi, K., Pentheroudakis, G., 2016. Non-epithelial ovarian cancer: Elucidating uncommon gynaecological malignancies. Anticancer Res. 36, 5031-5042. https://doi.org/10.21873/anticanres.11072

Horta, M., Cunha, T.M., 2015. Sex cord-stromal tumors of the ovary: A comprehensive review and update for radiologists. Diagnostic Interv. Radiol. 21, 277-286. https://doi.org/10.5152/dir.2015.34414

Jeong, Y.Y., Outwater, E.K., Kang, H.K., 2000. From the RSNA refresher courses: Imaging evaluation of ovarian masses. Radiographics 20, 1445-1470. https://doi.org/10.1148/radiographics.20.5.g00se101445

Forstner, R., Meissnitzer, M., Cunha, T.M., 2016. Update on Imaging of Ovarian Cancer. Curr. Radiol. Rep. 4, 1-11. https://doi.org/10.1007/s40134-016-0157-9 